\title{
Benzene adsorbed on metals: Concerted effect of covalency and van der Waals bonding
}

\author{
Wei Liu, ${ }^{1}$ Javier Carrasco, ${ }^{2}$ Biswajit Santra, ${ }^{1,3}$ Angelos Michaelides, ${ }^{4}$ Matthias Scheffler, ${ }^{1}$ and Alexandre Tkatchenko ${ }^{1, *}$ \\ ${ }^{1}$ Fritz-Haber-Institut der Max-Planck-Gesellschaft, Faradayweg 4-6, D-14195 Berlin, Germany \\ ${ }^{2}$ Instituto de Catálisis y Petroleoquímica, CSIC, Marie Curie 2, E-28049 Madrid, Spain \\ ${ }^{3}$ Department of Chemistry, Princeton University, Princeton, New Jersey 08544, USA \\ ${ }^{4}$ Thomas Young Centre, London Centre for Nanotechnology and Department of Chemistry, University College London, \\ London WC1E 6BT, United Kingdom
}

(Received 19 June 2012; revised manuscript received 12 November 2012; published 5 December 2012)

\begin{abstract}
The adsorption of aromatic molecules on metal surfaces plays a key role in condensed matter physics and functional materials. Depending on the strength of the interaction between the molecule and the surface, the binding is typically classified as either physisorption or chemisorption. Van der Waals (vdW) interactions contribute significantly to the binding in physisorbed systems, but the role of the vdW energy in chemisorbed systems remains unclear. Here we study the interaction of benzene with the (111) surface of transition metals, ranging from weak adsorption ( $\mathrm{Ag}$ and $\mathrm{Au}$ ) to strong adsorption ( $\mathrm{Pt}, \mathrm{Pd}, \mathrm{Ir}$, and $\mathrm{Rh}$ ). When vdW interactions are accurately accounted for, the barrier to adsorption predicted by standard density-functional theory (DFT) calculations essentially vanishes, producing a metastable precursor state on Pt and Ir surfaces. Notably, vdW forces contribute more to the binding of covalently bonded benzene than they do when benzene is physisorbed. Comparison to experimental data demonstrates that some of the recently developed methods for including $\mathrm{vdW}$ interactions in DFT allow quantitative treatment of both weakly and strongly adsorbed aromatic molecules on metal surfaces, extending the already excellent performance found for molecules in the gas phase.
\end{abstract}

DOI: 10.1103/PhysRevB.86.245405

PACS number(s): 68.43.-h, 31.15.E-, 31.15.V-, 71.15.Ap

\section{INTRODUCTION}

The adsorption of aromatic molecules at transition-metal surfaces is important for fundamental and applied surface science studies, ${ }^{1-3}$ and these systems show promise as components in (opto)-electronic devices. ${ }^{4}$ In the case of weak overlap of electron orbitals between the adsorbate and the substrate surface, the ubiquitous van der Waals (vdW) interactions are frequently the only force that binds the molecule to the surface. This situation is typically referred to as physisorption. In the chemisorption case, the covalent or ionic bonding dominates and the effect of vdW interactions on the overall strength of adsorption is typically assumed to be weak. In this study, we challenge this conventional view by demonstrating the significantly larger contribution of vdW energy to the stabilization of strongly adsorbed benzene on (111) surfaces of $\mathrm{Pt}, \mathrm{Pd}, \mathrm{Rh}$, and Ir metals when compared to physisorption on $\mathrm{Ag}(111)$ and $\mathrm{Au}(111)$ surfaces.

Whereas the role of vdW forces in the binding of atoms and molecules in the gas phase is reasonably well understood, at solid surfaces our understanding remains far from complete. Indeed, until recent developments (see, e.g., Refs. 5-8) for efficiently incorporating the long-range vdW energy within density-functional theory (DFT) calculations, it was not possible to determine the role of the vdW energy for extended systems and adsorption processes. ${ }^{9-11}$ A large majority of previous theoretical work on vdW interactions mainly focused on weakly bound systems. ${ }^{12-18}$ Typical examples include benzene (Bz) adsorbed on the $\mathrm{Ag}(111)$ and $\mathrm{Au}(111)$ surfaces, ${ }^{15-18}$ and noble gases on the $\mathrm{Cu}(111), \operatorname{Ag}(111), \operatorname{Pt}(111)$, and $\operatorname{Pd}(111)$ surfaces. ${ }^{10,19-21}$ A unifying aspect of these studies is the observation that the inclusion of $\mathrm{vdW}$ interactions into standard DFT within the generalized gradient approximation (GGA) often brings a large increase in binding, and results in a much better agreement with experimental adsorption distances and energies. However, the vdW forces can also have a qualitative impact on the adsorption process. One particularly interesting example was reported by Blügel's group, showing that the vdW forces are the key ingredient to trigger the binding of pyridine on $\mathrm{Cu}(110)$ from physisorption to weak chemisorption. ${ }^{22}$ Mittendorfer et $a .^{23}$ reported a novel mechanism for graphene adsorption on $\mathrm{Ni}(111)$, where weak covalent and vdW interactions lead to two different minima in the binding curve. Similar results were found by $\mathrm{Li}$ et al. ${ }^{24}$ Another example was shown in our recent work on the isophorone molecule $\left(\mathrm{C}_{9} \mathrm{H}_{14} \mathrm{O}\right)$ at the $\mathrm{Pd}(111)$ surface, which illustrated that the binding structure and the dehydrogenation pathway in this system can be predicted only after accounting for vdW interactions. ${ }^{25}$ The vdW interactions were also shown to play a role in the chemisorption of benzene on the $\mathrm{Si}(100)$ surface. ${ }^{26,27}$ However, in this case the vdW-DF method leads to a smaller adsorption energy than the pure Perdew-BurkeErnzerhof (PBE) functional, ${ }^{26}$ while the PBE + vdW method predicts a vdW contribution of $0.5 \mathrm{eV}^{27}$

In this work, we demonstrate the significant concerted effect of covalent bonding and $\mathrm{vdW}$ interactions for benzene interacting with metal surfaces, leading to qualitative changes in the adsorption behavior when vdW interactions are accurately treated (see Fig. 2). In particular, our calculations predict a metastable precursor state for benzene on $\mathrm{Pt}(111)$ in agreement with the experimental findings, ${ }^{28}$ along with a peculiar "phase transition" behavior of the projected highest occupied and lowest unoccupied molecular orbital (HOMO and LUMO, respectively) occupations of the benzene molecule. Comparison to experimental data demonstrates that recently developed methods for including vdW interactions in $\mathrm{DFT}^{8,10}$ allow quantitative treatment of both weakly and strongly adsorbed aromatic molecules on metal surfaces, extending the already excellent performance found for gas-phase molecules. ${ }^{8,29}$ 


\section{COMPUTATIONAL METHOD}

We used two different vdW-inclusive approaches in the present work: a newly developed $\mathrm{PBE}+\mathrm{vdW}{ }^{\text {surf }}$ method, ${ }^{10}$ as implemented in the FHI-aims all-electron code ${ }^{30}$ and the optB88-vdW method, ${ }^{8}$ as implemented in the VASP code. ${ }^{31,32}$ The PBE $+v_{d W}$ surf approach includes screened vdW interactions (beyond the pairwise atom-atom approximation) to study adsorbates on surfaces by a synergetic linkage of the $\mathrm{PBE}+\mathrm{vdW}$ method $^{6}$ for intermolecular vdW interactions with the Lifshitz-Zaremba-Kohn theory ${ }^{33}$ for the dielectric screening within the metal substrate. While the PBE $+\mathrm{vdW}^{\text {surf }}$ approach leads to accurate results in the asymptotic limit by construction, it uses a short-range damping function with one adjusted parameter. The optB88-vdW method is a modified version of the vdW-DF functional, ${ }^{5}$ by using an empirically optimized optB88-like exchange functional. Both $\mathrm{PBE}+\mathrm{vdW} \mathrm{W}^{\text {surf }}$ and optB88-vdW methods can accurately describe intermolecular interactions with mean absolute relative errors on the order of $9 \%, 8,29$ compared to coupled-cluster dimer binding energies for the S22 molecular database. Less is known about the performance of these methods for solids and weakly adsorbed molecules on surfaces, although encouraging results have been reported for a few condensed matter systems. ${ }^{10,11,32,34}$ It is important to benchmark the newly developed methods on a wider set of condensed matter systems, especially because the PBE $+\mathrm{vdW}^{\text {surf }}$ and optB88vdW methods are based on very different approximations. For comparison purposes, calculations using the vdW-DF functional and its second version $(v d W-D F 2)^{35}$ were also carried out for the $\mathrm{Bz} / \mathrm{Pt}(111)$ and $\mathrm{Bz} / \mathrm{Au}(111)$ systems.

The FHI-aims code was employed for the PBE $+\mathrm{vdW}^{\text {surf }}$, $\mathrm{PBE}+\mathrm{vdW}, \mathrm{PBE},{ }^{36}$ and local-density approximation (LDA) calculations. We used the "tight" settings, including the standard numerical atom-centered orbitals (NAO) basis set "tier2" for $\mathrm{H}$ and $\mathrm{C}$, and "tier1" for transition metals. For all structural relaxations, we used a convergence criterion of $10^{-2} \mathrm{eV} \AA^{-1}$ for the maximum final force. Also a convergence criteria of $10^{-5}$ electrons for the electron density and $10^{-4} \mathrm{eV}$ for the total energy of the system were utilized for all computations. The scaled zeroth-order regular approximation (ZORA) was applied for treating relativistic effects. Using these settings, the accuracy in determining the binding energy and equilibrium distance is better than $0.01 \mathrm{eV}$ and $0.005 \AA$, respectively.

For bulk lattice constant calculations, we used a Monkhorst$\mathrm{Pack}^{37}$ grid of $16 \times 16 \times 16 k$-points. The lattice constants of the bulk metals have been obtained by using the BirchMurnaghan equation of state fit to DFT cohesive energy curves. $^{38}$ Using the respective lattice constants from each method, we built up six-layer slabs with a $(3 \times 3)$ unit cell, with no reconstruction of $\mathrm{Pt}(111)$ and $\mathrm{Au}(111)$. Each slab was separated by a $20 \AA$ vacuum. The vdW interactions between metal atoms were also considered when performing the relaxations. We constrained the bottom four metal layers while fully relaxing the molecule and the uppermost two metal layers during geometry relaxations. For slab calculations, we used a $6 \times 6 \times 1 k$-points mesh.

For the calculation of the binding curves, we changed the adsorption height $d$ of $\mathrm{Bz}$, which is evaluated relative to the position of the unrelaxed topmost metal layer. For each structure, we fixed the $z$ coordinates of the carbon backbone and the metal atoms in the bottommost four of the employed six-layer surface model.

The VASP code was employed for the optB88-vdW, vdWDF, and vdW-DF2 calculations. Inner electrons were replaced by the projector augmented wave (PAW) method, while the monoelectronic valence electrons were expanded in plane waves with an $E_{\text {cutoff }}=500 \mathrm{eV}$. For slab calculations, we used a $4 \times 4 \times 1 k$-points mesh. For the metal supercell, we used a $(3 \times 3)$ unit cell with six atomic layers (three bottom layers fixed to the corresponding bulk optimal position for each method). Dipole correction was applied along the direction perpendicular to the metal surface. Geometry optimizations were performed with a residual force threshold of $0.03 \mathrm{eV}^{-1}$.

\section{RESULTS}

The typical strongly bound $\mathrm{Bz} / \mathrm{Pt}(111)$ system [adsorption energy 1.57-1.91 eV (Ref. 28)] and the typical weakly bound $\mathrm{Bz} / \mathrm{Au}(111)$ system [adsorption energy $0.73-0.87 \mathrm{eV}$ (Ref. 39)] are used first to demonstrate our point. Accurate experimental data is available for both of these systems, enabling direct quantitative verification of our theoretical calculations. To demonstrate the differences in the adsorption mechanism, we explore the potential-energy surface (PES) for $\mathrm{Bz}$ on the $\mathrm{Pt}(111)$ and $\mathrm{Au}(111)$ surfaces. We place a single $\mathrm{Bz}$ molecule at the eight high-symmetry adsorption sites of the (111) metal surface, ${ }^{40}$ followed by geometry relaxation. The adsorption geometries and energies for $\mathrm{Bz}$ on $\mathrm{Au}(111)$ and $\operatorname{Pt}(111)$ at the preferable adsorption site are shown in Fig. 1 and Table I. Already here one can clearly distinguish the different nature of bonding for the adsorption of $\mathrm{Bz}$ on $\mathrm{Pt}(111)$ and $\mathrm{Au}(111)$. Irrespective of the functional used

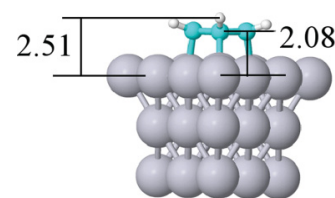

$\mathrm{Bz} / \mathrm{Pt}(111)$

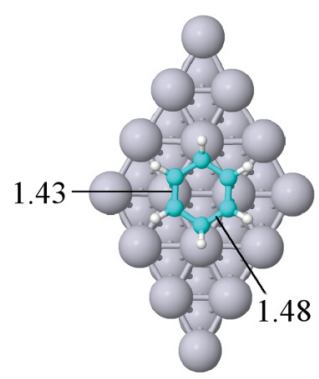

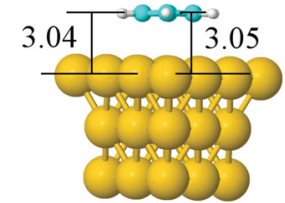

$\mathrm{Bz} / \mathrm{Au}(111)$

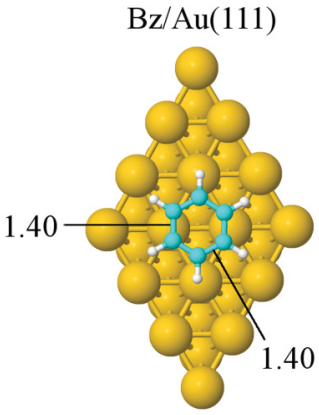

FIG. 1. (Color online) Adsorption structures of the Bz/Pt(111) system and $\mathrm{Bz} / \mathrm{Au}(111)$ system, both at the so-called bri30 adsorption site (see text). We carried out extended periodic calculations, but only a small part of the supercell is shown. Six metal layers were used but only the topmost three layers are depicted in the figure. The indicated distances $(\AA)$ are obtained based on the PBE $+v_{d W}^{\text {surf }}$ optimized structures. Gray, yellow, cyan, and white spheres represent $\mathrm{Pt}, \mathrm{Au}, \mathrm{C}$, and $\mathrm{H}$ atoms, respectively. Optimized lattice constants were used for every method. 
TABLE I. Comparison of adsorption energy $\left(E_{\mathrm{ad}}\right)$ and average perpendicular heights $\left(d_{\mathrm{CM}}\right.$ and $d_{\mathrm{HM}}$ for carbon-metal and hydrogenmetal, respectively) between DFT calculations and experimental data for $\mathrm{Bz}$ on $\mathrm{Pt}(111)$ and $\mathrm{Au}(111)$. The distances are referenced to the average positions of the relaxed topmost metal atoms. The adsorption energy $E_{\mathrm{ad}}$ is defined as $E_{\mathrm{ad}}=-\left(E_{\mathrm{AdSys}}-E_{\mathrm{Me}}-E_{\mathrm{Bz}}\right)$, where the subscripts $A d S y s, M e$, and $B z$ denote the adsorption system, the clean metal substrate, and the isolated Bz molecule, respectively.

\begin{tabular}{llccc}
\hline \hline System & \multicolumn{1}{c}{ Method } & $E_{\text {ad }}[\mathrm{eV}]$ & $d_{\mathrm{CM}}[\AA]$ & $d_{\mathrm{HM}}[\AA]$ \\
\hline Bz/Pt(111) & PBE+vdW surf & 1.96 & 2.08 & 2.51 \\
& optB88-vdW & 1.84 & 2.12 & 2.53 \\
& vdW-DF & 0.77 & 2.16 & 2.57 \\
& vdW-DF2 & 0.34 & 2.20 & 2.65 \\
& PBE & 0.81 & 2.10 & 2.54 \\
& LDA & 2.30 & 2.05 & 2.47 \\
& Experiment & $1.57-1.91^{\mathrm{a}}$ & $2.02 \pm 0.02^{\mathrm{b}}$ & \\
Bz/Au(111) & PBE+vdW & 0.74 & 3.05 & 3.04 \\
& optB88-vd & 0.79 & 3.23 & 3.23 \\
& vdW-DF & 0.59 & 3.44 & 3.42 \\
& vdW-DF2 & 0.56 & 3.29 & 3.27 \\
& PBE & 0.15 & 3.62 & 3.62 \\
& LDA & 0.49 & 2.83 & 2.82 \\
& Experiment & $0.73-0.87^{\mathrm{c}}$ & $2.95-3.10^{\mathrm{d}}$ & \\
\hline \hline
\end{tabular}

${ }^{\text {a Heat of adsorption measured with calorimetry, at the same coverage }}$ $(0.7 \mathrm{ML})$ used for the DFT calculations. ${ }^{28}$ The error estimates of $\pm 10 \%$ are taken from Ref. 28 . Recent work suggests reduced errors of $\pm 5 \% .^{48}$

${ }^{\mathrm{b}}$ LEED experiment. ${ }^{45}$

${ }^{\mathrm{c}}$ TPD experiment. ${ }^{10,39}$

${ }^{\mathrm{d}}$ Deduced data based on the experimental work function for $\mathrm{Bz}$ on $\mathrm{Au}(111)$ and adsorption distance for pentacene on $\mathrm{Au}(111) .{ }^{18,49,50}$

$\left(\mathrm{PBE}, \mathrm{PBE}+\mathrm{vdW}\right.$, and $\left.\mathrm{PBE}+\mathrm{vdW}^{\text {surf }}\right)$, the bri30 ${ }^{\circ}$ is the most preferable site for $\mathrm{Bz} / \mathrm{Pt}(111)$, with an angle of $30^{\circ}$ between the $\mathrm{C}-\mathrm{C}$ and $\mathrm{Pt}-\mathrm{Pt}$ bonds (see Fig. 1). This result is consistent with previous periodic slab GGA calculations, ${ }^{40-44}$ as well as low-energy electron diffraction (LEED) ${ }^{45}$ and scanning tunneling microscopy (STM) ${ }^{46}$ experiments. Moreover, the PES shows a corrugation of $1.33 \mathrm{eV}$ for $\mathrm{Bz} / \mathrm{Pt}(111)$ when using $\mathrm{PBE}+\mathrm{vdW}^{\text {surf }}$. In contrast, the PES for $\mathrm{Bz} / \mathrm{Au}(111)$ is found to be flat, with only $0.04 \mathrm{eV}$ corrugation. This result further justifies the STM observations that even at a temperature of $4 \mathrm{~K}, \mathrm{Bz}$ molecules are capable of diffusing over the $\mathrm{Au}(111)$ terraces. ${ }^{47}$

The analysis of the equilibrium distances and adsorption energies in Table I demonstrates that both $\mathrm{PBE}+\mathrm{vdW}^{\text {surf }}$ and optB88-vdW methods lead to an excellent agreement with the available experimental data. For the $\mathrm{Bz} / \mathrm{Pt}(111)$ system, the PBE $+\mathrm{vdW}^{\text {surf }}$ adsorption energy of $1.96 \mathrm{eV}$ is close to that from optB88-vdW $(1.84 \mathrm{eV})$, and both methods agree with the measured calorimetry values at $0.7 \mathrm{ML}$ (1.57-1.91 eV, the same coverage used for DFT calculations). ${ }^{28}$ The PBE $+\mathrm{vdW}^{\text {surf }}$ adsorption energy converges to $2.18 \mathrm{eV}$ with increasing surface cell size, within the error bar of calorimetry measurements in the limit of zero coverage $(1.84-2.25 \mathrm{eV}){ }^{28}$ Note that the exclusion of the $\mathrm{vdW}$ interactions in the strongly adsorbed $\mathrm{Bz} / \mathrm{Pt}(111)$ system would lead to a significant reduction in the binding energy (0.81 eV from PBE), in disagreement with the experimental data. The adsorption energies computed using the vdW-DF and vdW-DF2 methods are even smaller than those calculated with PBE. The adsorption energy for $\mathrm{Bz}$ adsorbed on the $\mathrm{Au}(111)$ surface is considerably smaller than that of $\mathrm{Bz} / \mathrm{Pt}(111)$. Also for $\mathrm{Bz} / \mathrm{Au}(111)$, the $\mathrm{PBE}+\mathrm{vdW}^{\text {surf }}$ adsorption energy $(0.74 \mathrm{eV})$ agrees very well with both the optB88-vdW result $(0.79 \mathrm{eV})$ and the experimental temperature-programmed desorption (TPD) data at $0.1 \mathrm{ML}(0.73-0.87 \mathrm{eV}) .{ }^{10,39} \mathrm{We}$ conclude that PBE $+v_{d W}{ }^{\text {surf }}$ and optB88-vdW methods yield quantitative agreement with experimental adsorption distances and energies for both weakly and strongly adsorbed $\mathrm{Bz}$ molecules. In contrast, LDA calculations are not systematic, underbinding for $\mathrm{Au}(111)$ and overbinding for $\mathrm{Pt}(111)$.

Deeper insight into the mechanism of $\mathrm{Bz}$ adsorption can be gained upon analysis of the binding energy curves, $E_{\text {ad }}(d)$, in Fig. 2. The binding-energy curves exhibit several characteristic effects. With decreasing distance the binding energy of the adsorbate system increases, determined mainly by vdW interactions, and here (for $d>3.5 \AA$ ) $\mathrm{Au}(111)$ and $\mathrm{Pt}(111)$ show very similar behavior. In both cases the calculations show a small broadening of the energy levels. The fully occupied $d$ band of $\mathrm{Au}$ is obviously stiffer than the partially empty $d$ band of Pt. In fact, for the latter the Pauli repulsion can be weakened by the rearrangement of $d$-electron density (a similar effect has been investigated in previous literature $\left.{ }^{19}\right)$. As a consequence, the $\mathrm{Bz}$ molecule gets closer to the surface of Pt and the HOMO and LUMO levels of the combined system broaden and hybridize noticeably. This goes together with significant electron transfer: The HOMO and HOMO- 1 orbitals of the Bz molecule get partially depleted and the LUMO and LUMO + 1 orbitals become partially filled. This behavior (broadening, shift, hybridization of levels, and electron transfer) is a clear signature of the covalent interaction for $\mathrm{Bz} / \mathrm{Pt}(111)$. Thus, at the adsorption geometry the wave function has attained a qualitatively new character. Figure 2 shows that this character change sets in for $\mathrm{Pt}$ at a distance of $3.1 \AA$. At $2.6 \AA$ nearly a full electron has been transferred from the HOMO and HOMO-1 levels to the LUMO and LUMO + 1 levels, and in the total energy we observe a "phase transition behavior" (cf. the peak at $2.6 \AA$ ). Finally, at the equilibrium geometry the electron transfer (rearrangement) is as large as $\sim 1.1$ electrons. For the Au surface the process is much weaker and, not surprisingly, a covalent contribution to the adsorption process remains negligible. Thus, the $\mathrm{vdW}$ attraction governs the interaction.

Further inspection of the electron density difference at the strongly bound minimum for $\mathrm{Bz} / \mathrm{Pt}(111)$ in Fig. 2(c) demonstrates the rather strong hybridization between the HOMO/LUMO orbitals of $\mathrm{Bz}$ and the $d_{z^{2}}$ orbitals of the $\mathrm{Pt}(111)$ atoms. For the same adsorption height, the electron density difference for $\mathrm{Bz} / \mathrm{Au}(111)$ is weak [see Fig. 2(d), inset]. The presence of two minima for $\mathrm{Bz} / \mathrm{Pt}(111)$ resembles the recently studied bonding of graphene on $\mathrm{Ni}(111) .^{23,24}$ However, the adsorption of $\mathrm{Bz}$ on $\mathrm{Pt}(111)$ exhibits a different feature. In fact, $\mathrm{Bz}$ is exothermically bound on $\mathrm{Pt}(111)$ already when using PBE without vdW interactions, while the PBE adsorption energy is endothermic for graphene on $\mathrm{Ni}(111)$. Evidently, the functionalization of aromatic molecules would allow control of the position and stability of the two adsorption minima on metallic surfaces. 

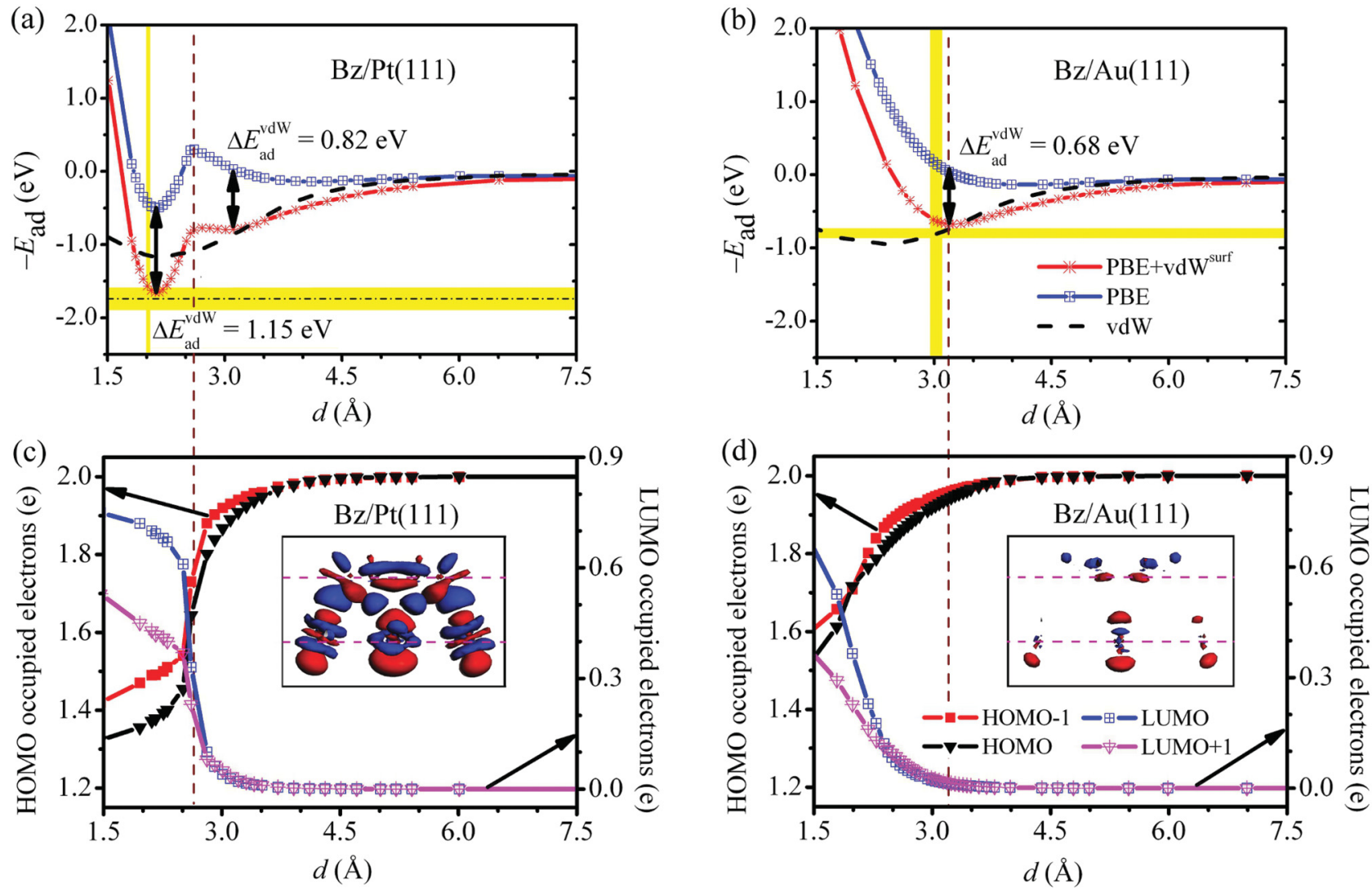

FIG. 2. (Color online) Top: Adsorption energy $-E_{\mathrm{ad}}$ as a function of the adsorption height $d$ for $\mathrm{Bz}$ on $\operatorname{Pt}(111)$ (a) and on $\mathrm{Au}(111)$ (b) from the PBE and PBE $+v d W^{\text {surf }}$ methods (the carbon backbone height $d$ from the surface is kept fixed). The experimental binding distances and adsorption energies are indicated by yellow intervals. Bottom: Integrated projected molecular density of states (Ref. 51) for the HOMO-1, HOMO, LUMO, and LUMO +1 orbitals of the benzene molecule as a function of $d$ for $\mathrm{Bz}$ on $\mathrm{Pt}(111)(\mathrm{c})$ and on $\mathrm{Au}(111)(\mathrm{d})$. The inset in panel (c) shows a side view of the electron density difference, which was obtained by subtracting the electron density of an isolated molecule and clean surface from an electron density plot of the entire adsorbed system upon Bz adsorption on $\operatorname{Pt}(111)$ at $d=2.08 \AA($ red $=\operatorname{electron}$ depletion, blue = electron accumulation). For the same value of the isosurface $\left(0.04 \AA^{-3}\right)$, the electron density difference for Bz/Au(111) at $d=2.08 \AA$ is significantly weaker, see the inset in panel (d).

Interestingly, while covalency is crucial for the $\mathrm{Bz} / \mathrm{Pt}(111)$ bonding character, energetically the $\mathrm{vdW}$ contribution is in fact significant. Upon inclusion of $\mathrm{vdW}$ interactions, the binding behavior is strongly modified - the barrier to adsorption vanishes, and a precursor physisorption state emerges for $\mathrm{Bz} / \mathrm{Pt}(111)$. The PBE $+\mathrm{vdW}^{\text {surf }}$ method lowers the adsorption energy from $0.50 \mathrm{eV}$ (pure PBE value) to $1.65 \mathrm{eV}$ in Fig. 2. Thus the final adsorption results from a strongly concerted, synergistic effort. Upon comparing the binding curves for $\mathrm{Bz} / \mathrm{Pt}(111)$ and $\mathrm{Bz} / \mathrm{Au}(111)$, we see that the vdW contribution (due to $\mathrm{vdW}^{\text {surf }}$ ) for $\mathrm{Bz} / \mathrm{Pt}(111), 1.15 \mathrm{eV}$, is even stronger that that for $\mathrm{Bz} / \mathrm{Au}(111), 0.68 \mathrm{eV}$. The screened $\mathrm{Bz} /$ surface $C_{3}$ vdW coefficient is essentially the same for $\mathrm{Pt}(111)$ and $\mathrm{Au}(111)$ surfaces (2.17 and 2.02 hartree bohr $^{3}$, respectively). Therefore, we conclude that the larger contribution of the $\mathrm{vdW}$ energy in the case of covalent bonding comes from the rather short adsorption distance of the Bz molecule from the surface.

Our conclusions hold in general for the adsorption of $\mathrm{Bz}$ on other transition metal surfaces (see Table II). For Bz/Ir(111), the binding curve shows the same characteristic features as for $\mathrm{Bz} / \mathrm{Pt}(111)$ in Fig. 2. For $\mathrm{Bz}$ adsorbed on the $\mathrm{Pd}(111)$, $\mathrm{Rh}(111)$, and $\operatorname{Ir}(111)$ surfaces, the vdW energy contributions from the $\mathrm{PBE}+\mathrm{vdW}^{\text {surf }}$ method are in the range of 0.97 $1.21 \mathrm{eV}$, greater than those for $\mathrm{Bz}$ physisorbed on $\mathrm{Ag}(111)$ and $\mathrm{Au}(111)(0.68-0.82 \mathrm{eV})$. Even larger vdW energies are found in more complex polyaromatic adsorption systems. For instance, the vdW energy is determined to be $1.77 \mathrm{eV}$ for naphthalene $\left(\mathrm{C}_{10} \mathrm{H}_{8}\right)$ on the $\mathrm{Pt}(111)$ surface with a $(5 \times 4)$ unit cell. Also for this case the calculated adsorption energy from $\mathrm{PBE}+\mathrm{vdW}^{\text {surf }}(2.91 \mathrm{eV})$ is within the experimental error bars $(2.80-3.42 \mathrm{eV}) .{ }^{52}$ For anthracene $\left(\mathrm{C}_{14} \mathrm{H}_{10}\right)$ on the $\mathrm{Pt}(111)$ surface with a $(6 \times 4)$ unit cell, the adsorption energy contributed by vdW interactions $(2.42 \mathrm{eV})$ largely exceeds that determined from the PBE functional $(1.38 \mathrm{eV})$.

TABLE II. Adsorption energies $E_{\mathrm{ad}}(\mathrm{eV})$ of Bz adsorbed on (111) surfaces of $\mathrm{Ag}, \mathrm{Pd}, \mathrm{Rh}$, and Ir.

\begin{tabular}{lccc}
\hline \hline System & PBE & PBE + vdW ${ }^{\text {surf }}$ & optB88-vdW \\
\hline Bz/Ag(111) & 0.09 & 0.75 & 0.72 \\
Bz/Pd(111) & 1.17 & 2.14 & 1.91 \\
Bz/Rh(111) & 1.48 & 2.52 & 2.27 \\
Bz/Ir(111) & 1.10 & 2.24 & 2.09 \\
\hline \hline
\end{tabular}




\section{CONCLUSION}

In summary, we have demonstrated that the inclusion of $\mathrm{vdW}$ interactions qualitatively changes the adsorption behavior for benzene strongly interacting with (111) metal surfaces. The vdW energy in $\mathrm{Bz} / \mathrm{Pt}(111)$, a typical strongly adsorbed system, is almost $0.5 \mathrm{eV}$ greater than that in $\mathrm{Bz} / \mathrm{Au}(111)$, a typical physisorbed system. The bonding mechanism of $\mathrm{Bz} / \mathrm{Pt}(111)$ stems from a synergistic effort of covalent bonding and vdW interactions, and it is characterized by a peculiar "phase transition" behavior in the projected HOMO/LUMO occupations of the benzene molecule. Our findings for $\mathrm{Bz}$ adsorbed on $\mathrm{Ag}, \mathrm{Au}, \mathrm{Pt}, \mathrm{Pd}, \mathrm{Rh}$, and Ir surfaces indicate that DFT calculations with dispersion interactions are essential for both weakly and strongly bound molecules on surfaces.

\section{ACKNOWLEDGMENTS}

We are grateful for support from the FP7 Marie Curie Actions of the European Commission, via the Initial Training Network SMALL (MCITN-238804). W.L. was funded by a fellowship from the Alexander von Humboldt Foundation. A.T. acknowledges support from the European Research Council (ERC Starting Grant VDW-CMAT). A.M. is supported by the ERC (ERC Starting Grant QUANTUMCRASS) and by the Royal Society through a Wolfson Research Merit Award. J.C. is a Ramón y Cajal fellow supported by the Spanish Government. *tkatchenko@fhi-berlin.mpg.de

${ }^{1}$ F. S. Tautz, Prog. Surf. Sci. 82, 479 (2007).

${ }^{2}$ P. Gomez-Romero, Adv. Mater. 13, 163 (2001).

${ }^{3}$ S. J. Jenkins, Proc. R. Soc. A 465, 2949 (2009).

${ }^{4}$ L. Kronik and N. Koch, MRS Bull. 35, 417 (2010).

${ }^{5}$ M. Dion, H. Rydberg, E. Schröder, D. C. Langreth, and B. I. Lundqvist, Phys. Rev. Lett. 92, 246401 (2004).

${ }^{6}$ A. Tkatchenko and M. Scheffler, Phys. Rev. Lett. 102, 073005 (2009).

${ }^{7}$ S. Grimme, J. Antony, S. Ehrlich, and H. Krieg, J. Chem. Phys. 132, 154104 (2010).

${ }^{8}$ J. Klimeš, D. R. Bowler, and A. Michaelides, J. Phys.: Condens. Matter 22, 022201 (2010).

${ }^{9}$ A. Tkatchenko, L. Romaner, O. T. Hofmann, E. Zojer, C. AmbroschDraxl, and M. Scheffler, MRS Bull. 35, 435 (2010).

${ }^{10}$ V. G. Ruiz, W. Liu, E. Zojer, M. Scheffler, and A. Tkatchenko, Phys. Rev. Lett. 108, 146103 (2012).

${ }^{11}$ J. Carrasco, B. Santra, J. Klimeš, and A. Michaelides, Phys. Rev. Lett. 106, 026101 (2011).

${ }^{12}$ C. Busse, P. Lazić, R. Djemour, J. Coraux, T. Gerber, N. Atodiresei, V. Caciuc, R. Brako, A. T. N'Diaye, S. Blügel, J. Zegenhagen, and T. Michely, Phys. Rev. Lett. 107, 036101 (2011).

${ }^{13}$ I. Hamada and M. Tsukada, Phys. Rev. B 83, 245437 (2011).

${ }^{14}$ A. K. Kelkkanen, B. I. Lundqvist, and J. K. Nørskov, Phys. Rev. B 83, 113401 (2011).

${ }^{15}$ M. Vanin, J. J. Mortensen, A. K. Kelkkanen, J. M. Garcia-Lastra, K. S. Thygesen, and K. W. Jacobsen, Phys. Rev. B 81, 081408 (2010).

${ }^{16}$ K. Toyoda, I. Hamada, K. Lee, S. Yanagisawa, and Y. Morikawa, J. Chem. Phys. 132, 134703 (2010).

${ }^{17}$ J. Wellendorff, A. Kelkkanen, J. J. Mortensen, B. I. Lundqvist, and T. Bligaard, Top. Catal. 53, 378 (2010).

${ }^{18}$ E. Abad, Y. J. Dappe, J. I. Martínez, F. Flores, and J. Ortega, J. Chem. Phys. 134, 044701 (2011).

${ }^{19}$ J. L. F. Da Silva, C. Stampfl, and M. Scheffler, Phys. Rev. Lett. 90, 066104 (2003).

${ }^{20}$ D. L. Chen, W. A. Al-Saidi, and J. K. Johnson, Phys. Rev. B 84, 241405 (2011).

${ }^{21}$ P. L. Silvestrelli, A. Ambrosetti, S. Grubisic, and F. Ancilotto, Phys. Rev. B 85, 165405 (2012).

${ }^{22}$ N. Atodiresei, V. Caciuc, P. Lazić, and S. Blügel, Phys. Rev. Lett. 102, 136809 (2009).

${ }^{23}$ F. Mittendorfer, A. Garhofer, J. Redinger, J. Klimeš, J. Harl, and G. Kresse, Phys. Rev. B 84, 201401 (2011).
${ }^{24}$ X. Li, J. Feng, E. Wang, S. Meng, J. Klimeš, and A. Michaelides, Phys. Rev. B 85, 085425 (2012).

${ }^{25}$ W. Liu, A. Savara, X. Ren, W. Ludwig, K.-H. Dostert, S. Schauermann, A. Tkatchenko, H.-J. Freund, and M. Scheffler, J. Phys. Chem. Lett. 3, 582 (2012).

${ }^{26}$ K. Johnston, J. Kleis, B. I. Lundqvist, and R. M. Nieminen, Phys. Rev. B 77, 121404 (2008).

${ }^{27}$ H.-J. Kim, A. Tkatchenko, J.-H. Cho, and M. Scheffler, Phys. Rev. B 85, 041403 (2012).

${ }^{28}$ H. Ihm, H. M. Ajo, J. M. Gottfried, P. Bera, and C. T. Campbell, J. Phys. Chem. B 108, 14627 (2004).

${ }^{29}$ A. Tkatchenko, R. A. DiStasio, Jr., R. Car, and M. Scheffler, Phys. Rev. Lett. 108, 236402 (2012).

${ }^{30}$ V. Blum, R. Gehrke, F. Hanke, P. Havu, V. Havu, X. Ren, K. Reuter, and M. Scheffler, Comput. Phys. Commun. 180, 2175 (2009).

${ }^{31}$ G. Kresse and J. Furthmüller, Phys. Rev. B 54, 11169 (1996).

${ }^{32}$ J. Klimeš, D. R. Bowler, and A. Michaelides, Phys. Rev. B 83, 195131 (2011).

${ }^{33}$ E. Zaremba and W. Kohn, Phys. Rev. B 13, 2270 (1976).

${ }^{34}$ G. X. Zhang, A. Tkatchenko, J. Paier, H. Appel, and M. Scheffler, Phys. Rev. Lett. 107, 245501 (2011).

${ }^{35}$ K. Lee, E. D. Murray, L. Kong, B. I. Lundqvist, and D. C. Langreth, Phys. Rev. B 82, 081101 (2010).

${ }^{36}$ J. Perdew, K. Burke, and M. Ernzerhof, Phys. Rev. Lett. 77, 3865 (1996).

${ }^{37}$ H. J. Monkhorst and J. D. Pack, Phys. Rev. B 13, 5188 (1976).

${ }^{38}$ F. Birch, Phys. Rev. 71, 809 (1947).

${ }^{39}$ D. Syomin, J. Kim, B. E. Koel, and G. B. Ellison, J. Phys. Chem. B 105, 8387 (2001).

${ }^{40}$ M. Saeys, M. F. Reyniers, G. B. Marin, and M. Neurock, J. Phys. Chem. B 106, 7489 (2002).

${ }^{41}$ M. Saeys, M. F. Reyniers, M. Neurock, and G. B. Marin, J. Phys. Chem. B 107, 3844 (2003).

${ }^{42}$ F. Mittendorfer, C. Thomazeau, P. Raybaud, and H. Toulhoat, J. Phys. Chem. B 107, 12287 (2003).

${ }^{43}$ C. Morin, D. Simon, and P. Sautet, J. Phys. Chem. B 108, 5653 (2004).

${ }^{44}$ W. Gao, W. Zheng, and Q. Jiang, J. Chem. Phys. 129, 164705 (2008).

${ }^{45}$ A. Wander, G. Held, R. Q. Hwang, G. S. Blackman, M. L. Xu, P. de Andres, M. A. Van Hove, and G. A. Somorjai, Surf. Sci. 249, 21 (1991). 
${ }^{46}$ P. S. Weiss and D. M. Eigler, Phys. Rev. Lett. 71, 3139 (1993).

${ }^{47}$ B. A. Mantooth, E. C. H. Sykes, P. Han, A. M. Moore, Z. J. Donhauser, V. H. Crespi, and P. S. Weiss, J. Phys. Chem. C 111, 6167 (2007).

${ }^{48}$ W. Lew, M. C. Crowe, E. Karp, O. Lytken, J. A. Farmer, L. Árnadóttir, C. Schoenbaum, and C. T. Campbell, J. Phys. Chem. C 115, 11586 (2011).
${ }^{49}$ K. Toyoda, Y. Nakano, I. Hamada, K. Lee, S. Yanagisawa, and Y. Morikawa, Surf. Sci. 603, 2912 (2009).

${ }^{50}$ E. Abad, J. Ortega, Y. J. Dappe, and F. Flores, Appl. Phys. A 95, 119 (2009).

${ }^{51}$ G. M. Rangger, L. Romaner, G. Heimel, and E. Zojer, Surf. Interface Anal. 40, 371 (2008).

${ }^{52}$ J. M. Gottfried, E. K. Vestergaard, P. Bera, and C. T. Campbell, J. Phys. Chem. B 110, 17539 (2006). 